\title{
Trigonometric Interpolation Kernel to Construct Deformable Shapes for User-Interactive Applications
}

\author{
Daniel Schmitter, Ricard Delgado-Gonzalo, and Michael Unser, Fellow, IEEE
}

\begin{abstract}
We present a new trigonometric basis function that is capable of perfectly reproducing circles, spheres and ellipsoids while at the same time being interpolatory. Such basis functions have the advantage that they allow to construct shapes through a sequence of control points that lie on their contour (2-D) or surface (3-D) which facilitates user-interaction, especially in 3-D. Our piecewise exponential basis function has finite support, which enables local control for shape modification. We derive and prove all the necessary properties of the kernel to represent shapes that can be smoothly deformed and show how idealized shapes such as ellipses and spheres can be constructed.
\end{abstract}

Index Terms-3-D shape representation, deformable model, piecewise exponential, splines.

\section{INTRODUCTION}

$\mathbf{S}$ HAPE representation and deformation is an ongoing research topic in the fields where shapes need to be constructed, visualized, approximated or segmented. Related research domains include shape modeling for industrial design [1]-[3], or segmentation in biomedical imaging [4]-[6], such as the design of active contour models [7]. Applications involving such modeling are often user-interactive allowing a user to modify the shape by directly interacting with it. Desirable properties of such models are summarized as follows: 1) Intuitive user-interaction: the shape must be deformable by letting a user to directly interact with its boundary in a simple manner; e.g. dragging with a computer mouse the contour of a curve or surface. 2) Local deformation: through user-interaction the shape should only deform in the neighborhood where the interaction takes place. 3) Smooth deformation: a small perturbation of the shape must result in a small deformation of the shape. 4) Reproduction of particular shapes: typically the model must be able to represent particular types of idealized shapes (e.g. polynomial curves, ellipses, spheres) and have good approximation properties. 5) Continuity: depending on the application it can be required that the shape be everywhere differentiable. 6) Numerically stable implementation: usually this requires that the underlying mathematical functions are well-defined. 7) Allowing fast optimization: in semi-automatic applications, opti-

Manuscript received June 10, 2015; revised July 24, 2015; accepted July 24, 2015. Date of publication July 28, 2015; date of current version July 31, 2015. This work was supported by the Swiss National Science Foundation under Grant 200020-144355. The associate editor coordinating the review of this manuscript an approving it for publication was Prof. Edmund Lam.

The authors are with the Biomedical Imaging Group, École polytechnique fédérale de Lausanne (EPFL), Lausanne, Switzerland.

Color versions of one or more of the figures in this paper are available online at http://ieeexplore.ieee.org.

Digital Object Identifier 10.1109/LSP.2015.2461557 mization schemes related to shape deformation might need to be included in the model.

Because it is not always possible to satisfy all of the constraints, in practice usually a trade-off between the above mentioned requirements needs to be done. Existing methods can generally be categorized by either using a discrete or a continuous-domain model. Discrete approaches use polygonal meshes [9], [10] or subdivision-based models [11]-[13] to represent shapes. They show high flexibility, but require a large amount of parameters for shape modeling, which is a drawback in optimization schemes. On the other hand, continuous-domain models are mostly based on Bézier curves [16], [17], spherical harmonics [18] or on compactly supported basis functions such as B-splines [19]-[23] which have an explicit analytical expression. However, B-splines are only able to represent polynomial shapes [24], [25] and therefore, do not allow for the construction of ellipses and spheres.

We present a new trigonometric basis function that enables the parametric representation and deformation of idealized shapes, such as ellipses and spheres. It allows to generate shape models that meet all of the requirements listed above and is particularly useful for simplified user-interaction because it is compactly supported and verifies the interpolation property. This means that the control points of the shape, which are accessible to the user, directly lie on its boundary.

The main contributions of this article are the derivation of the proposed interpolation kernel together with all the necessary properties to construct deformable models for shape representation. The motivation behind this work is the construction of 2-D [26], [7] and 3-D [27]-[30] active contour models for the segmentation of sphere-like structures in biomedical images such as roundish cells [30] or organs [31] (Fig. 1).

\section{PARAMETRIC SHAPE REPRESENTATION}

\section{A. Planar 2-D Curves}

We consider 2-D curves in the plane that are described by two coordinate functions $x(t)$ and $y(t)$ with $t \in \mathbb{R}$. It is possible to parameterize these coordinate functions by a suitable linear combination of integer-shifted basis functions, which are derived from a so-called generator $\varphi$. Such a parametric model represents a 2-D curve as

$$
\boldsymbol{r}(t)=\left(\begin{array}{l}
x(t) \\
y(t)
\end{array}\right)=\sum_{k \in \mathbb{Z}} \boldsymbol{c}[k] \varphi(t-k),
$$

where the $\left\{c[k]=\left(c_{x}[k], c_{y}[k]\right)\right\}_{k \in \mathbb{Z}}$ are called the control points. If $\varphi$ is of compact (i.e., finite) support the curve can be modfied locally by changing the position of a control point. 

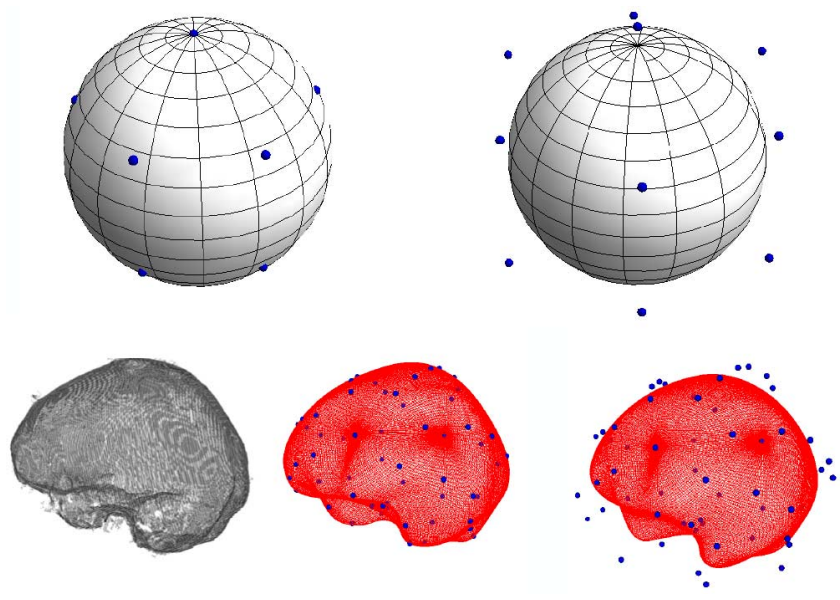

Fig. 1. Reproduction and approximation of shapes. Top row: Reproduction of the exact sphere using the proposed interpolatory (left) and the non-interpolatory function (right) from [8]. Bottom row: Segmentation of a brain volume. On the left a rendering of a brain is shown that has been extracted from a 3-D MRI scan as described in [31]. The 3-D brain structure has been segmented with a deformable model using our interpolatory (middle) as well as the non-interpolatory (right) basis function [8]. In both cases the results are $\mathcal{C}^{1}$-diffeomorphic to the sphere, which has been used to initialize them. The blue dots are the control points. They directly lie on the shape boundary for the interpolatory scheme. The segmented brain shape on the bottom right shows that user-interactive shape modification is difficult and non-intuitive for the non-interpolatory case, because it is unclear which part of the surface is affected by moving a control point.

The shapes that $\boldsymbol{r}$ can adopt (e.g. polynomial, elliptic) depend on the properties of the generator.

\section{B. Tensor-Product Surfaces}

The model (1) is extended to 3-D in order to construct surfaces that can be represented by a separable parameterization. In this case a surface $\boldsymbol{\sigma}$ is parameterized by $u, v \in \mathbb{R}$ as

$$
\begin{aligned}
\boldsymbol{\sigma}(u, v) & =\left(\begin{array}{l}
x(u, v) \\
y(u, v) \\
z(u, v)
\end{array}\right)=\left(\begin{array}{c}
x_{1}(u) \cdot x_{2}(v) \\
y_{1}(u) \cdot y_{2}(v) \\
z_{1}(u) \cdot z_{2}(v)
\end{array}\right) \\
& =\sum_{k \in \mathbb{Z}} \boldsymbol{c}_{1}[k] \varphi_{1}(u-k) \cdot \sum_{l \in \mathbb{Z}} \boldsymbol{c}_{2}[l] \varphi_{2}(v-l) \\
& =\sum_{k \in \mathbb{Z}} \sum_{l \in \mathbb{Z}} \underbrace{\boldsymbol{c}_{1}[k] \cdot \boldsymbol{c}_{2}[l]}_{\boldsymbol{c}[k, l]} \varphi_{1}(u-k) \varphi_{2}(v-l) .
\end{aligned}
$$

Displacing the control points in (1) and (2) respectively results in a continuous deformation of the corresponding shape.

\section{CONSTRUCTION OF THE INTERPOLATOLR}

In [8], we have proposed ellipse-reproducing basis functions that are defined as $\psi_{M}(t)=\sum_{n=0}^{2} \lambda_{n} \frac{\mathrm{d}^{n}}{\mathrm{~d} t^{n}} \beta_{\boldsymbol{\alpha}_{1}}(t)$, where $M$ is the number of control points used to construct a given function and $\beta_{\boldsymbol{\alpha}_{1}}$ is the 3-th order causal exponential B-spline defined in the Fourier domain as $\hat{\beta}_{\boldsymbol{\alpha}_{1}}(\omega)=\prod_{k=1}^{3} \frac{1-e^{\alpha_{k}-j \omega}}{j \omega-\alpha_{k}}$. Thereby, the vector $\boldsymbol{\alpha}_{1}=\left(0, \frac{j 2 \pi}{M},-\frac{j 2 \pi}{M}\right)$ specifies the poles of the B-spline. We have shown that $\psi_{M}$ is of minimal support and can be either smooth or interpolatory but not both at the same time. Finite support is important to implement fast optimization schemes [32].

\section{A. Proposed Interpolator}

Definition: Our piecewise exponential basis function $\phi_{M}$ is expressed in its causal form as

$$
\phi_{M}=\beta_{\boldsymbol{\alpha}_{1}} * \phi_{M}^{0},
$$

where $\phi_{M}^{0}=\gamma_{1}(M) \beta_{(0)}+\gamma_{2}(M)(\delta+\delta(\cdot-1))$ is a smoothing kernel of unit support, $\beta_{(0)}$ is the zeroth-degree B-spline, and $\gamma_{1}, \gamma_{2}$ are chosen such that the centered generator $\phi_{M}(\cdot+a)$, satisfies the interpolation condition

$$
\phi_{M}(k+a)=\delta_{k} .
$$

Here, $a$ is the appropriate shifting constant to center the generator, the dot in the argument of a function is a placeholder for its parameter, and $\delta_{k}$ is the Kronecker delta. Because $\beta_{\boldsymbol{\alpha}_{1}}$ is of support equal to 3 and $\phi_{M}^{0}$ has unit support, the resulting support of $\phi_{M}$ is equal to 4 and hence $a=2$. The unique weights $\gamma_{1}$ and $\gamma_{2}$ used to compute $\phi_{M}^{0}$ in (3) are computed by solving (4) while additionally enforcing $\phi_{M}$ to be symmetric, i.e., $\phi_{M}(t+a)=\phi_{M}(-t+a)$; a property that is especially convenient in practice. We find

$$
\gamma_{1}(M)=\frac{\pi^{3} \sec ^{2}\left(\frac{\pi}{M}\right)}{M^{2}\left(M \tan \left(\frac{\pi}{M}\right)-\pi\right)}
$$

and

$$
\gamma_{2}(M)=\frac{\pi^{2} \csc \left(\frac{\pi}{M}\right) \csc \left(\frac{2 \pi}{M}\right)\left(M-2 \pi \csc \left(\frac{2 \pi}{M}\right)\right)}{M^{2}\left(M \sec \left(\frac{\pi}{M}\right)-\pi \csc \left(\frac{\pi}{M}\right)\right)} .
$$

The explicit expression of the proposed interpolator $\varphi_{M}(t)=$ $\phi_{M}(t+a)$ is given by (5), shown at the bottom of the page, where the interpolator $\varphi_{M}$ is the centered (i.e., shifted) version

$$
\varphi_{M}(t)=\left(\begin{array}{ccc}
\frac{\sin ^{2}\left(\frac{\pi}{M}\right)\left(M \sin \left(\frac{2 \pi(t-2)}{M}\right)-2 \pi(t-2)\right)+\left(M \sin \left(\frac{2 \pi}{M}\right)-2 \pi\right) \sin ^{2}\left(\frac{\pi(t-2)}{M}\right)}{2\left(\cos \left(\frac{2 \pi}{M}\right)-1\right)\left(-M \sin \left(\frac{2 \pi}{M}\right)+\pi \cos \left(\frac{2 \pi}{M}\right)+\pi\right)} & 1 \leq t \leq 2 \\
\frac{M\left(\sin \left(\frac{2 \pi(t-2)}{M}\right)-2 \sin \left(\frac{2 \pi(t-1)}{M}\right)+\sin \left(\frac{2 \pi(t+1)}{M}\right)+\sin \left(\frac{2 \pi}{M}\right)-\sin \left(\frac{4 \pi}{M}\right)\right)+2 \pi t \cos \left(\frac{2 \pi}{M}\right)-2 \pi(t-1) \cos \left(\frac{4 \pi}{M}\right)-2 \pi \cos \left(\frac{2 \pi t}{M}\right)}{4\left(\cos \left(\frac{2 \pi}{M}\right)-1\right)\left(-M \sin \left(\frac{2 \pi}{M}\right)+\pi \cos \left(\frac{2 \pi}{M}\right)+\pi\right)} & 0 \leq t \leq 1 \\
\frac{M\left(\sin \left(\frac{2 \pi(t-1)}{M}\right)-2 \sin \left(\frac{2 \pi(t+1)}{M}\right)+\sin \left(\frac{2 \pi(t+2)}{M}\right)-\sin \left(\frac{2 \pi}{M}\right)+\sin \left(\frac{4 \pi}{M}\right)\right)+2 \pi\left(t \cos \left(\frac{2 \pi}{M}\right)-(t+1) \cos \left(\frac{4 \pi}{M}\right)+\cos \left(\frac{2 \pi t}{M}\right)\right)}{4\left(\cos \left(\frac{2 \pi}{M}\right)-1\right)\left(-M \sin \left(\frac{2 \pi}{M}\right)+\pi \cos \left(\frac{2 \pi}{M}\right)+\pi\right)} & -1 \leq t \leq 0 \\
\frac{\sin ^{2}\left(\frac{\pi}{M}\right)\left(2 \pi(t+2)-M \sin \left(\frac{2 \pi(t+2)}{M}\right)\right)+\left(M \sin \left(\frac{2 \pi}{M}\right)-2 \pi\right) \sin ^{2}\left(\frac{\pi(t+2)}{M}\right)}{2\left(\cos \left(\frac{2 \pi}{M}\right)-1\right)\left(-M \sin \left(\frac{2 \pi}{M}\right)+\pi \cos \left(\frac{2 \pi}{M}\right)+\pi\right)} & -2 \leq t \leq-1 \\
0 \quad & |t| \geq 2
\end{array}\right.
$$


of the causal generator $\phi_{M}$. In the following we derive the properties of the proposed interpolator that are useful in practical applications.

\section{B. Reproduction of Trigonometric Functions}

To be able to represent shapes that are deformations and translations of ellipses or spheres our generator $\varphi_{M}$ must reproduce sines and cosines, as well as constants.

Proposition 1: The interpolatory basis function $\varphi_{M}$ reproduces constants as well as $\cos \left(\frac{2 \pi \cdot}{M}\right)$ and $\sin \left(\frac{2 \pi \cdot}{M}\right)$ independently of the number of control points $M \geq 3$.

This result follows from [33, Proposition 2], where it was shown that reproduction properties are preserved through convolution. In [8] it was shown that $\beta_{\boldsymbol{\alpha}_{1}}$ reproduces the functions stated in Proposition 1 if and only if $M \geq 3$ and hence, by (3) $\varphi_{M}$ inherits from $\beta_{\boldsymbol{\alpha}_{1}}$ its ellipse-reproducing properties.

\section{Smoothness and Regularity}

A fundamental requirement for the construction of the basis function is that it must be everywhere differentiable. This is important w.r.t. shape deformation in order to avoid discontinuities when perturbing an idealy reproduced shape, such as an ellipse or sphere.

Proposition 2: The interpolator $\varphi_{M}$ belongs to $\mathcal{C}^{1}$ and has bounded second derivatives.

Proof: We re-express (3) in terms of exponential B-splines of different order as

$$
\begin{aligned}
\phi_{M} & =\beta_{\boldsymbol{\alpha}_{1}} * \phi_{M}^{0} \\
& =\beta_{\boldsymbol{\alpha}_{1}} *\left(\gamma_{1}(M) \beta_{(0)}+\gamma_{2}(M)(\delta+\delta(\cdot-1))\right) \\
& =\gamma_{1}(M) \beta_{\boldsymbol{\alpha}_{1} \cup(0)}+\gamma_{2}(M)\left(\beta_{\boldsymbol{\alpha}_{1}}+\beta_{\boldsymbol{\alpha}_{1}}(\cdot-1)\right),
\end{aligned}
$$

where we have used the property that the convolution between two exponential B-splines specified by $\beta_{\boldsymbol{\alpha}_{m}}$ and $\beta_{\boldsymbol{\alpha}_{n}}$ yields another exponential B-spline specified by $\boldsymbol{\alpha}=\boldsymbol{\alpha}_{m} \cup \boldsymbol{\alpha}_{n}$, i.e., the union of the sets of poles defining the functions to be convolved. Now we use the fact that the order $N$ of an exponential B-spline corresponds to the number of poles defining it. The number of times a B-spline is everywhere continuously differentiable is equal to $N-2$. From (6) we see that the lowest order of B-spline involved in the construction of $\varphi_{M}$ is $N=3$ and hence, due to the linearity of the derivative $\varphi_{M} \in \mathcal{C}^{1}$. The second part of Proposition 2 follows from the fact that (exponential) B-splines of order $N$ are Hölder-continuous of order $N-1$ with bounded derivatives [33].

\section{Convergence and Order of Approximation}

The order of approximation of the interpolator is of importance because it describes how fast an approximated function or shape converges towards the object being interpolated.

Proposition 3: The interpolator $\varphi_{M}$ converges to the (polynomial) Keys interpolator [34] (which in computer graphics is known as the Catmull-Rom spline [35]). It is given by

$$
\phi_{\mathrm{Keys}}=3 \beta^{3}-\left(\beta^{2}+\beta^{2}(\cdot-1)\right)
$$

and has an order of approximation of $L=3$. Thereby, $\beta^{3}$ and $\beta^{2}$ are the cubic and quadratic polynomial B-splines.

Proof: We observe that as $M$ grows large, i.e., $M \rightarrow \infty$ the 3 rd order exponential B-spline defined by $\boldsymbol{\alpha}_{1}$ converges to the 3rd order polynomial B-spline that is defined by its poles $\boldsymbol{\alpha}=(0,0,0)$. Since $\lim _{M \rightarrow+\infty} \gamma_{1}(M)=3$ and $\lim _{M \rightarrow+\infty} \gamma_{2}(M)=-1$ we obtain $\lim _{M \rightarrow+\infty} \varphi_{M}=\phi_{\text {Keys }}$.

Furthermore, $\varphi_{M}$ is able to approximate any curve with arbitrary precision by chosing $M$ sufficiently large [36].

\section{E. Riesz Basis}

It is desirable that the curves and surfaces given by (1) and (2) are uniquely specified by their sequence of control points $\{\boldsymbol{c}[k]\}_{k \in \mathbb{Z}}$ and $\{\boldsymbol{c}[k, l]\}_{k, l \in \mathbb{Z}}$ respectively. Therefore, the shifted basis functions $\left\{\varphi_{M}(\cdot-k)\right\}_{k \in \mathbb{Z}}$ should be linearly independent. Additionally, for practical reasons, the interpolation process must be numerically stable. These requirements are fullfilled if the generating function satisfies the Riesz-basis condition [33].

Proposition 4: For $M \geq 3$ the function $\varphi_{M}$ generates a Riesz basis, i.e., there exist two constants $0<A \leq B<\infty$, such that

$$
\begin{aligned}
0 & \leq A\|c\|_{l_{2}(\mathbb{Z})}^{2} \leq\left\|\sum_{k \in \mathbb{Z}} c[k] \varphi_{M}(\cdot-k)\right\|_{L_{2}(\mathbb{R})}^{2} \\
& \leq B\|c\|_{l_{2}(\mathbb{Z})}^{2} \leq \infty
\end{aligned}
$$

for all $c \in l_{2}(\mathbb{Z})$.

Due to space constraints we only outline a sketch of proof. It is based on the fact that (8) is expressed in the Fourier domain as $A \leq \sum_{k=-\infty}^{\infty}\left|\hat{\varphi}_{M}(\omega+2 \pi k)\right|^{2} \leq B$, and that using (6) the Fourier transform of $\varphi_{M}$ is given by $\hat{\varphi}_{M}(\omega)=\gamma_{1} \hat{\beta}_{\boldsymbol{\alpha}_{1} \cup(0)}(\omega)+$ $\gamma_{2}\left(\hat{\beta}_{\boldsymbol{\alpha}_{1}}\right)(\omega)\left(1+e^{-j \omega}\right)$. The complete proof is similar to the one of [37, Theorem 6.2].

\section{F. Affine Invariance and Partition of Unity}

The basis function must reproduce shapes irrespective of their orientation and position, i.e., they must be invariant to affine transformations. The following proposition formalizes this property for the 2-D case. The extension to 3-D is straightforward.

Proposition 5: The curves described by (1) are affine invariant, i.e.,

$$
\boldsymbol{A r}(t)+\boldsymbol{b}=\sum_{k \in \mathbb{Z}}(\boldsymbol{A c}[k]+\boldsymbol{b}) \varphi_{M}(t-k),
$$

where $\boldsymbol{A}$ is a $2 \times 2$ matrix and $\boldsymbol{b}$ is a $2-\mathrm{D}$ vector.

From (9) we see that affine invariance holds if and only if $\sum_{k \in \mathbb{Z}} \varphi_{M}(t-k)=1$, which is called partition of unity. Furthermore, from Proposition 1 we know that $\varphi_{M}$ reproduces constants. As a consequence and using the fact that $\varphi_{M}$ is an interpolator the partition of unity is verified and hence, also affine invariance. 


\section{REPRODUCTION OF ELLIPSES}

A direct consequence of Proposition 1 is that $\varphi_{M}$ allows us to construct ellipses independently from the number of control points $M \geq 3$. In this section we explicitly show how ellipses can be reproduced using the proposed basis functions. Because ellipses can be constructed by applying an affine transformation to a circle and using the property of our model to be affine invariant it suffices to show that we can generate circles.

Proposition 6: Using the generator $\varphi_{M}$ the unit circle is parametrized as

$$
\boldsymbol{r}(t)=\left(\begin{array}{c}
\cos (2 \pi t) \\
\sin (2 \pi t)
\end{array}\right)=\sum_{k=0}^{M-1}\left(\begin{array}{c}
c_{M}^{c}[k] \\
c_{M}^{s}[k]
\end{array}\right) \varphi_{M, \text { per }}(M t-k)
$$

where $\varphi_{M, \text { per }}=\sum_{n=-\infty}^{+\infty} \varphi_{M}(t-M n-k)$ is the $M$-periodization of $\varphi_{M}, c_{M}^{c}[\cdot]=\cos \left[\frac{2 \pi \cdot}{M}\right], c_{M}^{s}[\cdot]=\sin \left[\frac{2 \pi \cdot}{M}\right]$, and $t \in[0,1)$.

Proof: Using Proposition 1 combining with the fact that $\varphi_{M}$ is an interpolator and $\cos \left(\frac{2 \pi t}{M}\right)$ is $M$-periodic we write

$$
\begin{aligned}
& \cos \left(\frac{2 \pi t}{M}\right)=\sum_{k \in \mathbb{Z}} \cos \left[\frac{2 \pi k}{M}\right] \varphi_{M}(t-k) \\
& =\sum_{k=0}^{M-1} \sum_{n=-\infty}^{+\infty} \cos \left[\frac{2 \pi(M n+k)}{M}\right] \varphi_{M}(t-M n-k) \\
& =\sum_{k=0}^{M-1} \cos \left[\frac{2 \pi k}{M}\right] \underbrace{\sum_{n=-\infty}^{+\infty} \varphi_{M}(t-M n-k)}_{\varphi_{M, \operatorname{per}}(t-k)} \\
& \Rightarrow \cos (2 \pi t)=\sum_{k=0}^{M-1} \varphi_{M, \text { per }}(M t-k) .
\end{aligned}
$$

Combining (11) with a similar derivation for $\sin \left(\frac{2 \pi t}{M}\right)$ proves the claim.

Plots of the reconstructed trigonometric functions are shown in Fig. 2 as well as the unit circle that has been reconstructed by $\varphi_{M}$ with the smallest possible number of control points $M=3$ (Riesz-basis condition).

\section{REPRODUCTION OF SPHERES}

In this section, we outline our proposed construction of the sphere. Its parameterization as a tensor-product surface (2) results as a corollary from Proposition 6.

Corollary 1: Using the generator $\varphi_{M}$ the unit sphere is parametrized as

$$
\begin{aligned}
\boldsymbol{\sigma}(u, v)= & \left(\begin{array}{c}
x(u, v) \\
y(u, v) \\
z(u, v)
\end{array}\right)=\left(\begin{array}{c}
\cos (2 \pi u) \sin (\pi v) \\
\sin (2 \pi u) \sin (\pi v) \\
\cos (\pi v)
\end{array}\right) \\
= & \sum_{k=0}^{M_{1}-1} \sum_{l=-1}^{M_{2}+1} \boldsymbol{c}[k, l] \varphi_{M_{1}, \mathrm{per}}\left(M_{1} u-k\right) \\
& \times \varphi_{2 M_{2}}\left(M_{2} v-l\right)
\end{aligned}
$$
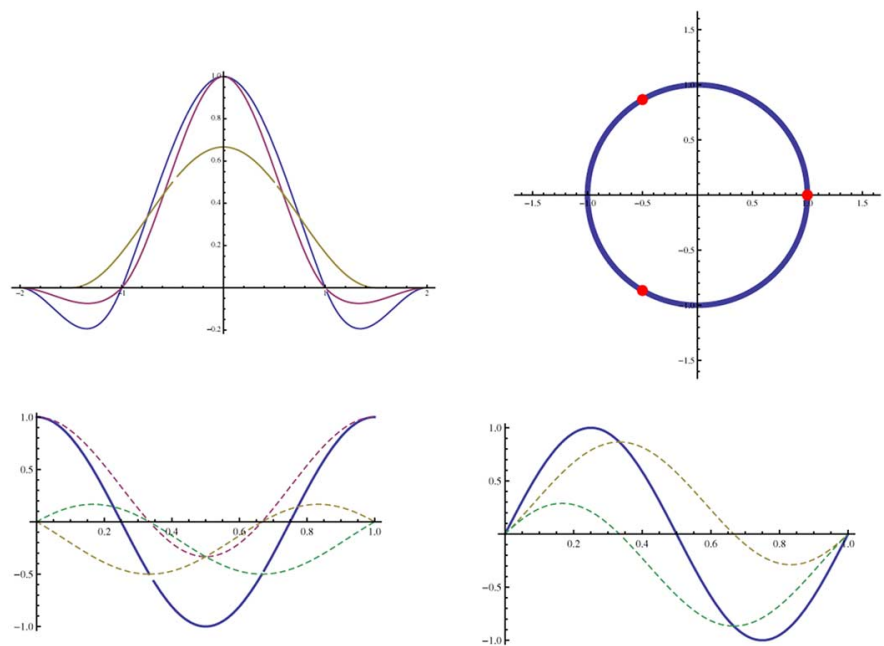

Fig. 2. Top left: The proposed trigonometric interpolator (blue), Keys interpolator (purple) and the non-interpolatory ellipse reproducing basis function from [8] (yellow). Top right: the circle obtained with the parametric equation $\boldsymbol{r}(t)=(\cos (2 \pi t), \sin (2 \pi t))$. Bottom: $\cos (2 \pi t)$ (left) and $\sin (2 \pi t)$ (right) are shown together with the basis functions (colored dashed lines) corresponding to $c_{M}^{c}[k]$ and $c_{M}^{s}[k]$, for $M=3$ and $k \in[0 \ldots M-1]$ respectively.

where $u, v \in[0,1)$, and the control points are given by

$$
\boldsymbol{c}[k, l]=\left(\begin{array}{c}
c_{M_{1}}^{c}[k] c_{2 M_{2}}^{s}[l] \\
c_{M_{1}}^{s}[k] c_{2 M_{2}}^{s}[l] \\
c_{2 M_{2}}^{c}[l]
\end{array}\right)
$$

The limits of the second sum in (12) are due to the fact that $v \in[0,1)$ and the support of $\varphi_{2 M_{2}}$ is limited to the interval $[-2,2]$. Therefore, we have $l \notin\left[-1, \ldots, M_{2}+1\right] \Leftrightarrow$ $\varphi_{2 M_{2}}\left(M_{2} v-l\right)=0$. The other terms in (12) follow from inserting $M=2 M_{2}$ in (11). Plots of a reconstructed sphere and a deformation of it are shown in Fig. 1, as well as a comparison with shapes constructed with the non-interpolatory basis function from [8].

\section{CONCLUSION}

We present a new trigonometric kernel to construct deformable shape models. We show that the kernel satisfies the necessary requirements needed for their construction such as: interpolation condition, compact support, smoothness, reproduction properties, Riesz-basis condition. The main advantage of the proposed basis functions is that they are smooth while also being interpolatory, therefore allowing the control points of a constructed shape to lie directly on the shape boundary; a feature that allows for intuitive shape manipulation in user-interactive applications. We explicitly show how to construct idealized shapes such as circles and spheres. We illustrate the use of such models in demo-videos showing 2-D and 3-D user-interactive deformable models that are constructed using (10) and (12). They are avialble at http://bigwww.epfl.ch/demo/interpolated-shapes/. 


\section{REFERENCES}

[1] M. Audette, A. Chernikov, and N. Chrisochoides, "A review of mesh generation for medical simulators," in Handbook of Real-World Applications in Modeling and Simulation. Hoboken, NJ, USA: Wiley, 2012, vol. 2, p. 261.

[2] A. Garg, A. Sageman-Furnas, B. Deng, Y. Yue, E. Grinspun, M. Pauly, and M. Wardetzky, "Wire mesh design," ACM Trans. Graph., vol. 33, no. 4, pp. 66:1-66:12, 2014.

[3] B. Deng, S. Bouaziz, M. Deuss, A. Kaspar, Y. Schwartzburg, and M. Pauly, "Interactive design exploration for constrained meshes," Comput.-Aided Design, vol. 61, pp. 13-23, 2015.

[4] T. McInerney and D. Terzopoulos, "Deformable models in medical image analysis: A survey," Medical Image Analysis, vol. 1, no. 2, pp. 91-108, 1996.

[5] T. Heimann and H.-P. Meinzer, "Statistical shape models for $3 \mathrm{~d}$ medical image segmentation: A review," Med. Image Anal., vol. 13, no. 4, pp. 543-563, 2009.

[6] A. Dufour, L. Tzu-Yu, C. Ducroz, R. Tournemenne, B. Cummings, R. Thibeaux, N. Guillen, A. Hero, and J.-C. Olivo-Marin, "Signal processing challenges in quantitative 3-d cell morphology: More than meets the eye," IEEE Signal Process. Mag., vol. 32, no. 1, pp. 30-40, Jan. 2015.

[7] R. Delgado-Gonzalo, V. Uhlmann, D. Schmitter, and M. Unser, "Snakes on a plan: A perfect snap for bioimage analysis," IEEE Signal Process. Mag., vol. 32, no. 1, pp. 41-48, Jan. 2015.

[8] R. Delgado-Gonzalo, P. Thévenaz, and M. Unser, "Exponential splines and minimal-support bases for curve representation," Comput. Aided Geom. Design, vol. 29, no. 2, pp. 109-128, Feb. 2012.

[9] M. Botsch, S. Steinberg, S. Bischoff, and L. Kobbelt, "Openmesh: A generic and efficient polygon mesh data structure," in Proc. OpenSG Symp, Darmstadt, Germany, 2002.

[10] M. Botsch, L. Kobbelt, M. Pauly, P. Alliez, and B. Levy, Polygon Mesh Processing. Natick, MA, USA: A K Peters, 2010.

[11] N. Dyn and E. Farkhi, "Spline subdivision schemes for compact sets. A survey," Serdica Math. J., vol. 28, pp. 349-360, 2002.

[12] M. Charina, C. Conti, and L. Romani, "Reproduction of exponential polynomials by multivariate non-stationary subdivision schemes with a general dilation matrix," Numer. Math., vol. 127, no. 2, pp. 223-254, 2014.

[13] P. Novara and L. Romani, "Building blocks for designing arbitrarily smooth subdivision schemes with conic precision," J. Computat. Appl. Math., vol. 279, pp. 67-79, 2015.

[14] K. Conti and C. Hormann, "Polynomial reproduction for univariate subdivision schemes of any arity," J. Approx. Theory, vol. 163, no. 4, pp. 413-437, 2011.

[15] M. Charina and C. Conti, "Polynomial reproduction of multivariate scalar subdivision schemes," J. Computat. Appl. Math., vol. 240, no. 0 , pp. 51-61, 2013.

[16] H. Prautzsch, W. Boehm, and M. Paluszny, Bézier and B-Spline Techniques. New York, NY, USA: Springer-Verlag, 2002.

[17] L. Romani and M. Sabin, "The conversion matrix between uniform B-spline and Bézier representations," Comput. Aided Geom. Design, vol. 21, no. 6, pp. 549-560, 2004.

[18] C. Brechbuehler, G. Gerig, and O. Kuebler, "Parametrization of closed surfaces for 3-d shape description," Comput. Vis. Image Understand., vol. 61 , no. 2 , pp. 154-170, 1995.
[19] L. L. Schumaker, Spline functions: basic theory, ser. Pure and Applied Mathematics. New York, NY, USA: Wiley, 1981.

[20] T. Lyche and L. Schumaker, "Local spline approximation methods," $J$. Approx. Theory, vol. 15, no. 4, pp. 294-325, 1975.

[21] T. Lyche, "Discrete cubic spline interpolation," BIT Numer. Math., vol. 16, no. 3, pp. 281-290, 1976.

[22] L. Piegl and W. Tiller, The NURBS Book, 2nd Ed. ed. Berlin, Germany: Springer, 2010

[23] C. Manni, F. Pelosi, and L. Sampoli, "Generalized B-splines as a tool in isogeometric analysis," Comput. Meth. Appl. Mech. Eng., vol. 200, no. 58, pp. 867-881, 2011.

[24] C. V. Beccari, G. Casciola, and L. Romani, "Construction and characterization of non-uniform local interpolating polynomial splines," $J$. Computat. Appl. Math., vol. 240, pp. 5-19, 2013.

[25] T. Dokken, T. Lyche, and K. Pettersen, "Polynomial splines over locally refined box-partitions," Comput. Aided Geom. Design, vol. 30, no. 3, pp. 331-356, 2013.

[26] M. Kass, A. Witkin, and D. Terzopoulos, "Snakes: Active contour models," Int. J. Comput. Vis., vol. 1, no. 4, pp. 321-331, Jan. 1987.

[27] G. Gerig, M. Styner, and G. Szekely, "Statistical shape models for segmentation and structural analysis," in Proc. 2002 IEEE Int. Symp. Biomedical Imaging, 2002, pp. 18-21.

[28] A. Dufour, R. Thibeaux, E. Labruyere, N. Guillen, and J.-C. OlivoMarin, "3-D Active meshes: Fast discrete deformable models for cell tracking in 3-D time-lapse microscopy," IEEE Trans. Image Process., vol. 20, no. 7, pp. 1925-1937, Jul. 2011.

[29] D. Barbosa, T. Dietenbeck, J. Schaerer, J. D’hooge, D. Friboulet, and O. Bernard, "B-Spline explicit active surfaces: An efficient framework for real-time 3-D region-based segmentation," IEEE Trans. Image Process., vol. 21, no. 1, pp. 241-251, Jan. 2012.

[30] R. Delgado-Gonzalo, N. Chenouard, and M. Unser, "Spline-based deforming ellipsoids for interactive 3d bioimage segmentation," IEEE Trans. Image Process., vol. 22, no. 10, pp. 3926-3940, Oct. 2013.

[31] D. Schmitter, R. Delgado-Gonzalo, G. Krueger, and M. Unser, "Atlas-free brain segmentation in 3D proton-density-like MRI images," in Proc. Eleventh IEEE Int. Symp. Biomedical Imaging: From Nano to Macro (ISBI'14), Beijing, China, Apr.-May 29-2, 2014, pp. 629-632.

[32] M. Jacob, T. Blu, and M. Unser, "Efficient energies and algorithms for parametric snakes," IEEE Trans. Image Process., vol. 13, no. 9, pp. 1231-1244, Sep. 2004.

[33] M. Unser and T. Blu, "Cardinal exponential splines: Part I Theory and filtering algorithms," IEEE Trans. Signal Process., vol. 53, no. 4, pp. 1425-1438, Apr. 2005.

[34] R. Keys, "Cubic convolution interpolation for digital image processing," IEEE Trans. Acoust., Speech, Signal Process., vol. 29, no. 6, pp. 1153-1160, Dec. 1981.

[35] E. Catmull and R. Rom, "A class of local interpolating splines," in Computer aided geometric design. New York, NY, USA: Academic, 1974, pp. 317-326.

[36] T. Blu and M. Unser, "Quantitative Fourier analysis of approximation techniques: Part I Interpolators and projectors," IEEE Trans. Signal Process., vol. 47, no. 10, pp. 2783-2795, Oct. 1999.

[37] M. Unser and P. Tafti, An Introduction to Sparse Stochastic Processes. Cambridge, U.K.: Cambridge Univ. Press, 2014. 